\title{
ENHANCED MICROWAVE ASSISTED PROCESSING OF 2D CYLINDRICAL POROUS FOOD DIELECTRIC
}

\author{
S. Kumari ${ }^{1}$, S. K. Samanta ${ }^{1}$, K. Patel ${ }^{1}$ \\ ${ }^{1}$ Indian Institute of Technology Patna, Bihta, Patna-801103, India \\ sushma.pcb16@iitp.ac.in; sksamanta@iitp.ac.in; kushpatel1407@gmail.com
}

\begin{abstract}
:
The importance of microwave assisted thermal processing for the food industry relies on the fact of the procedure being economical and swift along with ensuring high quality product. The sample has been assumed as a porous solid food with meat being the continuous phase and oil or water being the dispersed phase. The resultant matrix of meat-oil or meat-water is considered to be a 2D cylinder being subjected to microwave irradiation in two different interaction schemes i.e. lateral and radial. The thermal effect of the interaction is calculated by applying Galerkin finite element analysis to simultaneously solve the Maxwell's equation and heat transfer equation using pertinent boundary conditions. The preliminary solution gives the value of average power absorption at different sample sizes which exhibit few peak points depicting enhanced heating and power absorption. Further study consists of analyzing the spatial distribution of power and temperature along with the transient temperature difference and mean temperature evolution within the sample corresponding to designated observation points. The heating strategy exhibiting high heating rate and low thermal nonuniformity is recommended as the preferred optimum condition. For both meat-oil and meatwater porous solids, the radial irradiation is recommended as the optimal heating strategy corresponding to smaller sample diameter (OP: 1) whereas lateral irradiation is recommended for larger sample diameter (OP: 2). Overall the present work serves as a guide for enhanced microwave assisted heating of porous food samples in the shape of $2 \mathrm{D}$ cylinders.
\end{abstract}

Keywords: Microwave, Porous food, Optimum heating, Average power absorption

\section{Introduction:}

The electromagnetic waves with the wavelengths ranging from $1 \mathrm{~m}$ to $1 \mathrm{~mm}$ are known as microwaves and the typical frequency range is $300 \mathrm{MHz}$ to $300 \mathrm{GHz}$. The value of dielectric loss of a material is the determining factor in conversion of electrical energy into heat energy ${ }^{1-3}$. The volumetric heat generation effect of microwave has gained attention in different processing industries such as food, pharmaceutical, ceramics, polymer and many more $^{3-5}$. The exigencies of a swift, qualitative and economical procedure for the food industry was addressed by the microwave assisted processing such as heating, drying, thawing, sterilization, tempering, etc ${ }^{4,6,7}$.

The importance of proper investigation on processing of food prior to its industrial scaling is discussed by Karim and Hawlader (2005) where an analysis shows a loss of 30-40 \% of global food production in developing nations as a result of processing faults ${ }^{8}$. So, researchers are striving to come up with novel physics-based models to best imitate food samples. Ayappa et al. (1992) was the first to report a combined analysis of microwave and thermal transport for $1 \mathrm{D}$ and 2D solid samples ${ }^{9}$. The analysis and formulation for the occurrence of resonance at a specific sample size and dimension was formed by Ayappa et al. (1991) ${ }^{1}$. Basak and Ayappa (1997) performed thawing of solid samples observing higher melting rate during resonances at some specific thicknesses ${ }^{10}$. 
The mathematical imitation of a food is slightly difficult as food is amorphous and hygroscopic in nature and structurally non-homogenous ${ }^{11}$. A food material in itself is a complex sample with multiple components and varying dielectric properties which makes its mathematical modeling arduous. Many authors have come up with models but they tend to take assumptions to reduce mathematical complications. The most realistic characterization of food can be as a porous material with the dielectric response calculated as the operative dielectric properties of solid frame and the fluid present in the voids. Only a few studies have been reported on the heating effect of typical 1D porous-food dielectric with oil and air as dispersed medium using various metallic, ceramic or composite supports $^{7,12}$. A few 2D analyses of microwave heating of food samples studied till date have not considered the effect of porosity of the material ${ }^{4,13}$. Basak (2007) has carried out a detailed analysis to study the effect of lateral and radial irradiations on microwave heating of 2D solid meat cylinders and oil samples ${ }^{4}$. The effect of shape on the microwave heating dynamics of food materials was analyzed for different shapes like circular and square by Bhattacharya and Basak (2017) $)^{13}$.

Here we have carried out a detailed theoretical analysis on enhanced microwave heating of typical food samples (porous meat) in the form of 2D cylinders in the presence of lateral and radial irradiations. The food sample has been assumed to be a highly lossy porous dielectric with the meat as the continuous phase and oil or water as the dispersed phase of the porous solid structure. The resulting porous materials such as meat-oil and meat-water, where oil and water act as the fluid medium of the porous body, have been analyzed with operative dielectric properties for various porosity values $(\phi=0.1,0.25$ and 0.4$)$.

\section{Theoretical modeling and solution strategy:}

\section{Microwave propagation: Power and temperature formulations}

The extent of the absorption of microwave irradiation by the porous food dielectric has been analyzed through the electromagnetic field equation which assists the study of temperature distribution owing to different irradiation schemes i.e. lateral and radial. The scheme of lateral mode of irradiation assumes the sample to have unidirectional interaction (left in this study) due to being stationary in a uniform plane wave, whereas the radial irradiation scheme implies the sample to have a multidirectional interaction due to being rotated.

The electromagnetic field consists of mutually perpendicular electric and magnetic field with magnitude varying in the $\mathrm{z}$-direction i.e. the direction of wave propagation considered in this study (see Fig. 1). The propagation of microwave due to uniform electric field, $E_{x}$ varying only in the $y-z$ plane in a $2 \mathrm{D}$ porous solid can be given by Maxwell's equation as shown below ${ }^{1}$.

$$
\nabla^{2} E_{x}+\kappa^{2} E_{x}=0
$$

The propagation constant, $\kappa$, depends on dielectric constant $\kappa^{\prime}$, dielectric loss $\kappa^{\prime \prime}$ and frequency of electromagnetic wave, $f$, which can be given as:

$$
\kappa=2 \pi f\left(\sqrt{\kappa^{\prime}+i \kappa^{\prime \prime}}\right) / c
$$

The $c$ in the above equation is the speed of light.

The sample having a porous structure has been assumed to contain a dispersed phase and a continuous phase with both having different dielectric and thermal properties. The operative dielectric property of the sample can be evaluated by the Fricke's conductivity model as given below:

$\kappa_{o p}^{*}=\frac{\kappa_{c}^{*}\left[\kappa_{d}^{*}(1+a \phi)+a \kappa_{c}^{*}(1-\phi)\right]}{\kappa_{d}^{*}(1-\phi)+\kappa_{c}^{*}(a+\phi)}$ 
where, $\kappa_{c}^{*}$ and $\kappa_{d}^{*}$ are relative complex dielectric properties of the continuous and dispersed phases, respectively and are assumed as temperature independent. The value of ' $a$ ' is assumed to be 2 for the present case study which is due to the assumption of spherical dispersion.

Energy balance equation within the porous food sample due to microwave assisted heat source can be expressed as

$\rho C_{p} \frac{\partial T}{\partial t}=k \nabla^{2} T+P(z)$

where $\rho$, the operative density, $C_{p}$, the operative specific heat and $k$, the operative thermal conductivity can be expressed as operative properties such as:

$\rho=(1-\phi) \rho_{c}+\phi \rho_{d}$

$C_{p}=(1-\phi) C_{p c}+\phi C_{p d}$

and

$k=(1-\phi) k_{c}+\phi k_{d}$

The power per unit volume can be obtained by:

$$
P=\left(2 \pi f \varepsilon_{0} \kappa_{o p}^{\prime \prime}(\phi) E_{x} E_{x}^{*}\right) / 2
$$

where, $\varepsilon_{0}$ is permittivity of free space, $\phi$ is the porosity, $\kappa_{o p}^{\prime \prime}$ is the operative dielectric loss of the porous food and $E_{x}^{*}$ is the complex conjugate of the electric field. The above equation on being integrated over the entire domain considering $\mathrm{S}$ as the cross-sectional area and $\Gamma$ as the outer surface of the porous solid can be given as:

$P_{a v}=\frac{1}{S} \iint_{\Gamma} P(\Omega) d \Omega$

The insulation condition (no flux condition) has been used for outer surface as the boundary condition.
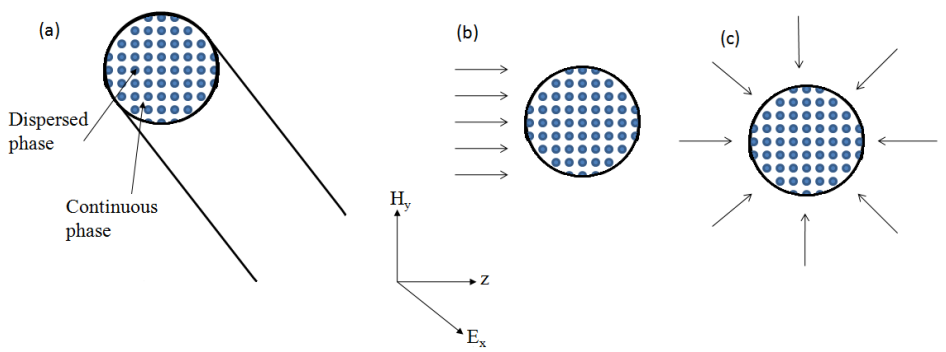

Fig. 1. Schematic illustrations of 2D porous food cylinder incident with (a) uniform plane waves, (b) lateral irradiation and (c) radial irradiation.

\section{Solution strategies}

The field equation given by Maxwell's and heat balance equation are solved simultaneously using Galerkin finite element method assuming appropriate boundary conditions $[1,7]$. The common element at the interface of the two phases satisfies the continuity condition of field variables and fluxes. To discretize the time domain, Crank-Nicholson method is used, and the nonlinear residual equations are solved using Newton Raphson method. The simulations are carried out for meat as the continuous phase and oil or water as dispersed phase of the porous cylinder considering the initial temperature as 
$275 \mathrm{~K}$, ambient temperature as $300 \mathrm{~K}$ and the microwave irradiation of $1,1.5$ and $2 \mathrm{~W} . \mathrm{cm}^{-2}$ intensities and $2450 \mathrm{MHz}$ frequency. The effective intensity of incident radiation from the source has been assumed to be identical for both the lateral and radial irradiation schemes.

\section{Results and discussion:}

A theoretical analysis has been performed to study the thermal effects on the 2D cylindrical porous food dielectrics subjected to microwave irradiation of $2450 \mathrm{MHz}$ frequency. The analysis comprises of studying the effect of different porosity conditions $(\phi=0.1,0.25$ and 0.4 ), different intensities $\left(I_{0}=1,1.5\right.$ and $\left.2 \mathrm{~W} . \mathrm{cm}^{-2}\right)$ and different dispersed phases (oil, water) on the heating strategy. The study of average power absorption within a porous cylindrical sample with respect to diameter of the sample gives a preliminary understanding of enhanced heating and power absorption within the sample. Owing to the main objective of comparing the heating strategy of both lateral and radial irradiation mode, the peaks obtained for either of the mode leads to selection of the sample size for both modes and being labeled as observation points (OP). The information on the average power absorption needs further exploration through the spatial distribution of power and temperature to understand the occurrence of resonances due to the constructive inferences of the transmitted and reflected waves. The exploration of distribution of heat inside the sample leads the way to the selection of the optimum heating strategy based on factor of high thermal uniformity and heating rate. The plots of average temperature and temperature difference with respect to time give the heating rate of the procedure and the extent of thermal non-uniformity or the thermal runaway, respectively. The heating strategy favoring low thermal non-uniformity and high heating rate is optimum. However, the decision is always inclined towards uniform distribution of temperature.

\section{Meat-oil porous dielectric at $\mathrm{I}_{0}=1 \mathrm{Wcm}^{-2}$ and $\phi=0.4$}

The microwave warming of porous meat-oil dielectric at frequency $f=2450 \mathrm{MHz}$, intensity $I_{0}=1 \mathrm{Wcm}^{-2}$ and porosity $\phi=0.4$ has been analysed with the aim of determining the best suited heating strategy. The plot of average power absorption with respect to the size of the sample exhibits the smaller samples to absorb more power corresponding to lateral irradiation mode and larger samples to absorb more power during radial irradiation mode. The peaks obtained due to constructive interferences of the waves are chosen as observation point (OP) for detailed analysis. The OP:1 and OP:2 are selected corresponding to sample diameter, $d_{c}=1.78$ and $3.00 \mathrm{~cm}$, respectively as shown in Fig. 2 . The OP: 1 corresponds to average power, $\mathrm{P}_{\mathrm{av}}=1.330$ and $0.940 \mathrm{~W} . \mathrm{cm}^{-3}$ for lateral and radial modes of irradiation, respectively. In addition, the average power absorption for OP: 2 exhibits the value of $\mathrm{P}_{\mathrm{av}}=$ 0.461 and $0.852 \mathrm{~W} . \mathrm{cm}^{-3}$ for lateral and radial schemes, respectively. It may also be noted that the effect of resonance dies down significantly for $d_{c} \geq 3 \mathrm{~cm}$ and the average power is found to decrease almost asymptotically for both lateral and radial irradiation cases. The inset of the plot includes the spatial distribution of power inside the sample. The sample at OP: 1 subjected to lateral irradiation displays two maxima in spatial power, one being 2.5 $\mathrm{W} . \mathrm{cm}^{-3}$ at the left side and the other being $3 \mathrm{~W} . \mathrm{cm}^{-3}$ at the right side whereas the radial irradiation displays a single central maxima of $2.5 \mathrm{~W} . \mathrm{cm}^{-3}$. OP: 2 displays single central maxima for both lateral and radial irradiation modes of 1 and $4 \mathrm{~W} . \mathrm{cm}^{-3}$, respectively. The thermal spatial distribution analysis at $\mathrm{t}=120$ seconds for meat-oil porous cylinders corresponding to lateral and radial irradiations at OP: 1 and OP: 2 have been displayed in Fig. 3(a). The maximum temperature for OP: 1 is observed at both the left and right faces and is around 350 and $390 \mathrm{~K}$, respectively for lateral irradiation. However, at the same OP, 
a central maxima of around $350 \mathrm{~K}$ for radial irradiation is observed. Moreover, for the OP: 2 , both the lateral and radial irradiations display a central maxima of 310 and $380 \mathrm{~K}$, respectively. This highlights the unpredictability of hotspot formation in the sample with different schemes and parameters. To further decide the optimum strategy, a study on the transient temperature difference and mean temperature evolution is performed.

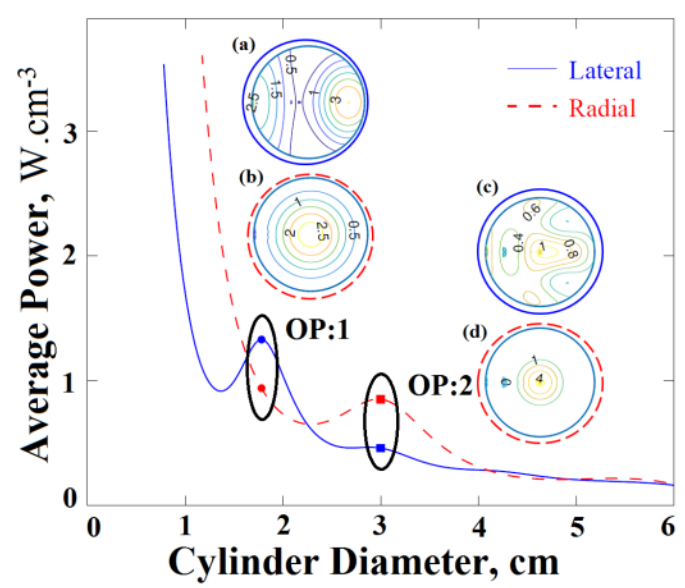

Fig. 2. Average power $\left(\mathrm{W} . \mathrm{cm}^{-3}\right)$ vs. cylinder diameter for various meat-oil samples due to lateral and radial irradiations corresponding to $\phi=0.4 ; f=2450 \mathrm{MHz} ; I_{0}=1 \mathrm{~W} . \mathrm{cm}^{-2}$. The inset is the representation of the power distribution inside the cylindrical sample (a) for lateral heating at $d_{c}=1.78 \mathrm{~cm}$, (b) for radial heating at $d_{c}=1.78 \mathrm{~cm},(\mathbf{c})$ for lateral heating at $d_{c}=$ $3.00 \mathrm{~cm}$ and (d) for radial heating at $\mathrm{d}_{\mathrm{c}}=3.00 \mathrm{~cm}$.
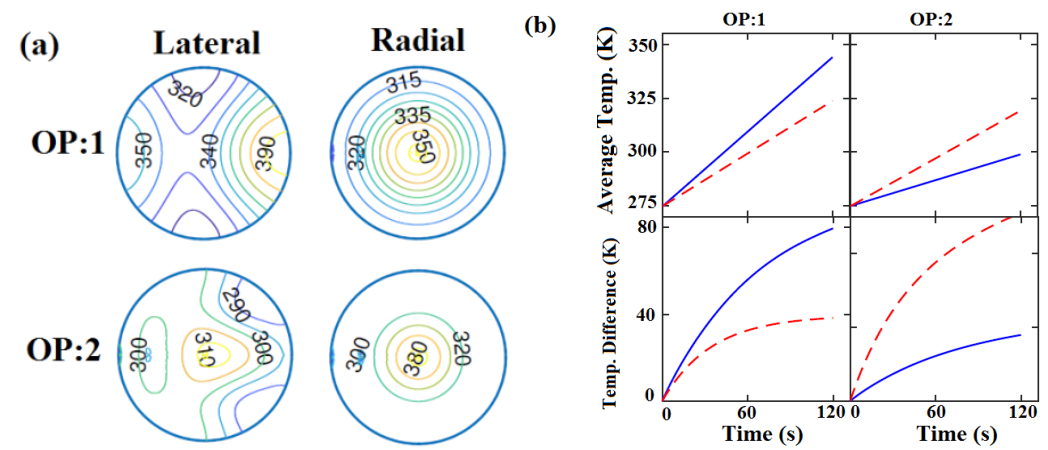

Fig. 3. (a) Spatial representation of thermal mapping inside the meat-oil food cylinders during microwave assisted warming at $\phi=0.4 ; f=2450 \mathrm{MHz} ; I_{0}=1 \mathrm{~W} . \mathrm{cm}^{-2}$ for 120 seconds.

(b) Average temperature $(\mathrm{K})$ in the meat-oil food cylinders vs. time (s) [upper panel];

Temperature difference $(\mathrm{K})$ in the meat-oil food cylinders vs. time (s) [lower panel].

The temperature difference vs. time which is a measure of the degree of non-uniformity of temperature distribution within the sample and average temperature vs. time that denotes the heating rate have been presented in the lower and upper panels of Fig. 3(b), respectively. An optimal heating strategy can be characterized as one with higher heating rate and smaller thermal runaway. At OP: 1, the heating rate is slightly higher for lateral irradiation however, 
the thermal non-uniformity is very large making the radial irradiation as the desired strategy. Although, at OP: 2 the lateral irradiation exhibits lower heating but lower thermal nonuniformity makes it the preferred heating strategy.

\section{Meat-water porous dielectric at $I_{0}=1.5 \mathrm{Wcm}^{-2}$ and $\phi=0.1$}

The analysis of microwave warming of porous meat-water food dielectric at a frequency of $2450 \mathrm{MHz}$, intensity $I_{0}=1.5 \mathrm{Wcm}^{-2}$ and porosity $\phi=0.1$ has been performed with the objective to determine the best heating strategy. Fig. 4 exhibits the average power absorption with respect to the sample diameter along with the spatial distribution of power at the chosen peaks labeled as OPs. The OP: 1 has been marked at sample diameter $d_{c}=1.30 \mathrm{~cm}$ with average power $\mathrm{P}_{\mathrm{av}}=2.736$ and $2.060 \mathrm{~W} \cdot \mathrm{cm}^{-3}$ for lateral and radial irradiation modes, respectively. The next OP i.e. OP: 2 for lateral and radial heating schemes is noted at $d_{c}=$ $2.12 \mathrm{~cm}$ with average power $\mathrm{P}_{\mathrm{av}}=0.920$ and $2.196 \mathrm{~W} . \mathrm{cm}^{-3}$, respectively.

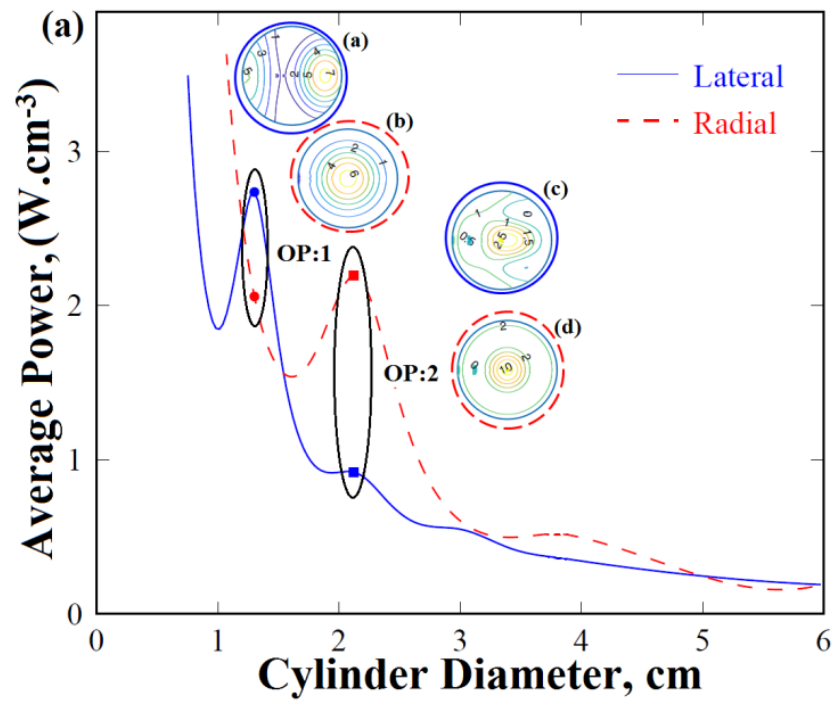

Fig. 4. Average power (W. $\left.\mathrm{cm}^{-3}\right)$ vs. cylinder diameter for various meat-water samples due to lateral and radial irradiations corresponding to $\phi=0.1 ; f=2450 \mathrm{MHz} ; I_{0}=1.5 \mathrm{~W} . \mathrm{cm}^{-2}$. The inset is the representation of the power distribution inside the cylindrical sample (a) for lateral heating at $\mathrm{d}_{\mathrm{c}}=1.30 \mathrm{~cm}$, (b) for radial heating at $\mathrm{d}_{\mathrm{c}}=1.30 \mathrm{~cm}$, (c) for lateral heating at $\mathrm{d}_{\mathrm{c}}=2.12 \mathrm{~cm}$ and (d) for radial heating at $\mathrm{d}_{\mathrm{c}}=2.12 \mathrm{~cm}$.

A detailed spatial distribution of temperature within the sample and study of the transient temperature difference and mean temperature evolution and distribution within the sample have also been performed for different OPs selected (see Fig. 5). The temperature contour in Fig. 5 (a) in the case of OP: 1 present a right sided maxima of $410 \mathrm{~K}$ for lateral irradiation and a central maxima of $370 \mathrm{~K}$ for radial irradiation. The OP: 2 displays a central maxima for both the irradiation modes with $320 \mathrm{~K}$ and $420 \mathrm{~K}$ for lateral and radial irradiations, respectively.

The investigation at OP: 1 for meat-water sample recommended the radial irradiation as the preferred heating strategy due to higher thermal uniformity even with slightly lower heating rate (see Fig.5 (b)). In addition, since OP: 2 exhibits lower heating rate but higher thermal 
uniformity for the lateral irradiation hence it is recommended as the preferred heating strategy.
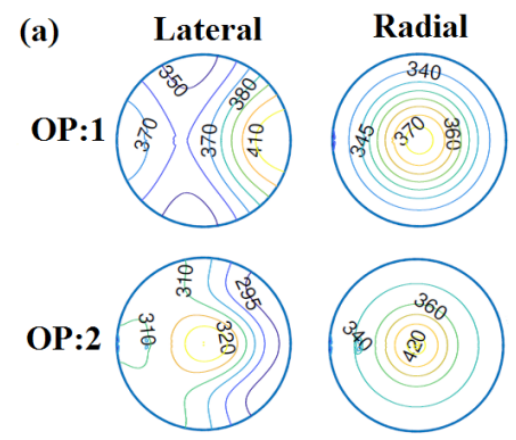

(b)

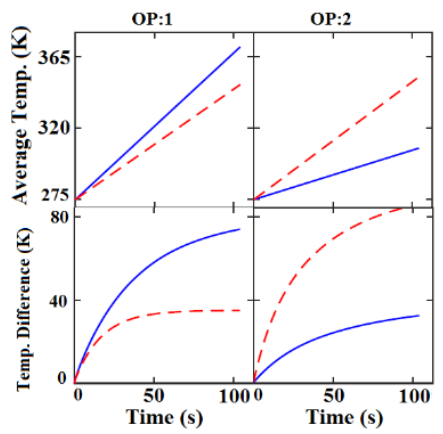

Fig 5. (a) Spatial representation of thermal mapping inside the meat-water food cylinders during microwave assisted warming at $\phi=0.1 ; f=2450 \mathrm{MHz} ; I_{0}=1.5 \mathrm{~W} . \mathrm{cm}^{-2}$ for 100 seconds. (b) Average temperature (K) in the meat-water food cylinders vs. time (s) [upper panel]; Temperature difference $(\mathrm{K})$ in the meat-water food cylinders vs. time (s) [lower panel].

Overall, the dielectric properties of the porous food samples and its dimension $\left(d_{c}\right)$ can be considered as two of the major parameters that are creating the diverse scenario of heating rate and thermal runaway within the samples. In addition, the final choice of lateral/radial irradiation is a trade-off between two parameters namely higher heating rate and minimal thermal runaway where minimal thermal runaway is generally given more emphasis owing to the importance of preservation of the quality of the sample. Similar work related to other food systems, porosity conditions and intensity of the microwave irradiation has also been carried out, however, results are not reported here for the brevity of the manuscript. The present analysis works as a guide for enhanced microwave assisted processing of 2D porous food dielectrics.

\section{Conclusions:}

An extensive analysis for investigating the thermal effect of microwave irradiation on 2D porous food cylinders with meat as continous phase and oil or water as dispersed phase has been perfomed. The analysis entails the importance of the role of lateral and radial irradiation schemes for obtaining an optimum heating strategy. Preliminarily, the extent of absorption of average power with respect to the sample size (cylinder diameter) has been studied for obtaining the power absorption and distribution within meat samples. Further, a detailed mathematical analysis on spatial temperature distributions and the transient temperature difference and mean temperature evolution within the sample for both the lateral and radial irradiation schemes has been carried out to decide an optimal heating strategy for any specific OP chosen from the precedent analysis. The plot of average temperature and temperature difference against time present us the heating rate and thermal non-uniformity, respectively. The recommended optimal heating strategies decide the procedure to have high heating rate with minimal thermal runaway (thermal non-uniformity).

Based on the recommendation for the optimal heating strategy for both meat-oil and meatwater porous solids, radial irradiation is recommended as the optimal heating strategy corresponding to smaller sample diameter (OP: 1) whereas lateral irradiation is recommended for larger sample diameter (OP: 2). Observing the overall scenarios, the choice of lateral/radial irradiations depends primarily on the sample dielectric properties and 
sample dimension $\left(d_{c}\right)$ and the suitability of any specific irradiation as an optimal heating strategy is measured based on the heating rate and thermal runaway for each case. Hence, the sample dielectric properties, sample dimension, non-uniformity in temperature distribution and heating rate come out to be the determining factors for efficient heating process for porous food dielectrics. Finally, the present study can be extended for other porous solids with different compositions of continuous and dispersed phases.

\section{References}

[1] Ayappa KG, Davis HT, Crapiste G, Davis EA, Gordon J. Chem Eng Sci. 1991;46:1005-16.

[2] Samanta SK, Basak T. Food Res Int. 2010;43:148-66.

[3] Samanta SK, Basak T. Food Res Int. 2009;42:1337-50.

[4] Basak T. Chem Eng Sci. 2007;62:3185-96.

[5] Verma P, Samanta SK. Environ Chem Lett. 2018;16:969-1007.

[6] Chandrasekaran S, Ramanathan S, Basak T. Food Res Int. 2013;52:243-61.

[7] Aparna K, Basak T, Balakrishnan AR. Int J Heat Mass Transf. 2007;50:3072-89.

[8] Karim MA, Hawlader MNA. Int J Heat Mass Transf. 2005;48:4914-25.

[9] Ayappa KG, Davis HT, Davis EA, Gordon J. Aiche J. 1992;38:1577-92.

[10] Basak T, Ayappa KG. Aiche J. 1997;43:1662-74.

[11] Khan MIH, Joardder MUH, Kumar C, Karim MA. Crit Rev Food Sci Nutr. 2016;57:1-19.

[12] Basak T, Aparna K, Meenakshi A, Balakrishnan AR. Int J Heat Mass Transf. 2006;49:4325-39.

[13] Bhattacharya M, Basak T. Innov Food Sci Emerg Technol. 2017;39:247-66. 